\title{
ON THE INFLUENCE OF DIFFUSION STABILIZATION IN OLDROYD-B FLUID FLOW SIMULATIONS
}

\author{
M. Pires ${ }^{1,2,3}$, T. Bodnár 3,4 \\ ${ }^{1}$ Department of Mathematics, Technology Sciences School, University of Évora, \\ Rua Romão Ramalho, 7000-671, Évora, Portugal \\ 2 CIMA-UE, CEMAT-IST \\ 3 Institute of Mathematics, Czech Academy of Sciences, \\ Žitná 25, 11567 Prague 1, Czech Republic \\ ${ }^{4}$ Faculty of Mechanical Engineering, Czech Technical University in Prague, \\ Karlovo Náměstí 13, 12135 Prague 2, Czech Republic
}

\begin{abstract}
This work presents some numerical tests of finite element solution of incompressible Oldroyd-B fluid flows. The effect of numerical stabilization using artificial stress diffusion is investigated in detail. The limits of Weissenberg number $\mathcal{W} e$ for which is possible to obtain the numerical solution were studied depending on the Reynolds number $\mathcal{R} e$ and the diffusion parameter. Series of numerical tests was performed for steady two-dimensional Oldroyd-B fluid flow in corrugated channel (tube). The numerical results clearly proved the advantage (higher attainable $\mathcal{W} e$ ) of stabilized numerical method over the classical formulation without the artificial stress diffusion.
\end{abstract}

Keywords: Finite Element Method, Oldroyd-B Fluid, Numerical Stabilization, Stres Diffusion.

\section{Introduction}

The numerical simulation of non-Newtonian viscoelastic fluids flow is a challenging problem. The need to solve a coupled system for fluid variables and associated stress tensor brings many computational as well as theoretical difficulties. One of the well known problems is the so called High Weissenberg Number Problem, i.e. the instability of the numerical solution for higher values of Weissenberg number. Such instability prevents the problem from being solved by standard numerical methods and techniques. There is a wide body of literature and number of approaches describing and trying to resolve this issue [1]-[4], [6], [12].

One of the approaches being often adopted in this situation is based on addition of stress diffusion term into the transport equations for viscoelastic stress tensor. Such extra term can be interpreted artificial diffusion applied to each stress component. There is however also physical, fluid microstructure based argument on addition of such term into the constitutive model. In any case, the additional term will certainly affect the solution of the problem and special care should be taken to keep the modified model consistent with the original problem [10], [11].

The modified stress diffusion technique adopted and tested here is slightly different. The steady solution is searched by solving an unsteady problem by a time-marching method, where the steady state is recovered for $t \rightarrow \infty$, subject to stationary boundary conditions. Instead of the classical addition of artificial stress diffusion term proportional to Laplacian of extra stress $\alpha \Delta \tau$, the term proportional to Laplacian of (pseudo) time derivative of extra stress $\alpha \Delta \boldsymbol{\tau}_{t}$ was used. This means, the additional term is only present during the transient phase and should vanish in when approaching the stationary case. The steady solution should not be affected by such vanishing artificial term, however the stability of the numerical method should be improved.

\section{Mathematical Model}

The unsteady, incompressible, isothermal viscoelastic flow of homogeneous Oldroyd-B fluid is considered in a bounded domain $\Omega \subset \mathbb{R}^{d}(d=2,3)$ over the time interval $\left[0, T_{f}\right]\left(T_{f}>0\right)$. The governing system of equations can be written in dimensionless form as: 


$$
\left\{\begin{array}{l}
\mathcal{R} e\left(\frac{\partial \boldsymbol{u}}{\partial t}+\boldsymbol{u} \cdot \nabla \boldsymbol{u}\right)+\nabla p=2(1-\eta) \nabla \cdot \mathbf{D}+\nabla \cdot \boldsymbol{\tau}+\mathbf{f} \\
\nabla \cdot \boldsymbol{u}=0 \\
\boldsymbol{\tau}+\mathcal{W} e\left(\frac{\partial \boldsymbol{\tau}}{\partial t}+\boldsymbol{u} \cdot \nabla \boldsymbol{\tau}-\nabla \boldsymbol{u}^{T} \cdot \boldsymbol{\tau}-\boldsymbol{\tau} \cdot \nabla \boldsymbol{u}\right)=2 \eta \mathbf{D}
\end{array}\right.
$$

where $\mathcal{R} e=\frac{\boldsymbol{U} L}{\nu}$ and $\mathcal{W} e=\frac{\lambda \boldsymbol{U}}{L}$ are the Reynolds and Weissenberg numbers, respectively, $\boldsymbol{\tau}$ is the viscoelastic contribution to the extra stress tensor given by $\mathbf{T}=2 \nu_{e} \mathbf{D}+\boldsymbol{\tau}, \mathbf{D}=\frac{1}{2}\left(\nabla u+\nabla u^{T}\right)$ is the strain tensor (symmetric part of velocity gradient), $\nu_{e}$ can either represent the viscosity of the solvent (for the polymer solution) or part of the total (apparent) stress viscosity of the system depending on the stress model adopted. Here $\eta$ is such that $\nu=\nu_{e}+\eta$, and $\nu$ is the total kinematic viscosity. The body force is denoted by $\mathbf{f}$.

\subsection{Variational formulation}

The numerical model presented in this paper is formulated on a bidimensional bounded domain $\Omega$, whose boundary is $\partial \Omega=\Gamma_{i n} \cup \Gamma_{w} \cup \Gamma_{\text {out }}$. The above (strong form of) the dimensionless Oldroyd-B model governing equations (without a body force) is supplemented by the initial and boundary conditions:

$$
\begin{cases}\boldsymbol{u}=0 & \text { on } \Gamma_{w} \\ \boldsymbol{u}=u_{i n} & \text { on } \Gamma_{i n} \\ \boldsymbol{\tau}=\boldsymbol{\tau}_{\text {in }} & \text { on } \Gamma_{i n} \\ \boldsymbol{u}_{\mid t=0}=u_{0} & \end{cases}
$$

The following function spaces are defined

$$
\begin{gathered}
\mathcal{V}=\left\{\mathbf{u} \in \mathbf{H}^{1}(\Omega): \mathbf{u}=0 \text { on } \Gamma_{w} \text { and } \mathbf{u}=u_{i n} \text { on } \Gamma_{i n}\right\}, \\
L_{0}^{2}(\Omega)=\left\{p \in L^{2}(\Omega): \int_{\Omega} p d \Omega=0\right\}, \\
\mathcal{S}=\left\{\mathbf{S} \in\left[L^{2}(\Omega)\right]^{2 \times 2}: \mathbf{S}^{T}=\mathbf{S}\right\} .
\end{gathered}
$$

The variational formulation of the problem (1-2), for initial data $u_{0} \in \mathcal{V}$ such that $\nabla \cdot u_{0}=0$, is:

Find $(\boldsymbol{u}, p, \boldsymbol{\tau}) \in L^{2}(0, T ; \mathcal{V}) \times L^{2}\left(0, T ; L_{0}^{2}(\Omega)\right) \times L^{2}(0, T ; \mathcal{S})$ such that

$$
\begin{cases}\int_{\Omega} 2(1-\eta) \mathbf{D}: \nabla \mathbf{v}+\mathcal{R} e \int_{\Omega}\left(\frac{\partial \boldsymbol{u}}{\partial t}+\boldsymbol{u} \cdot \nabla \boldsymbol{u}\right) \cdot \mathbf{v}-\int_{\Omega} p \nabla \cdot \mathbf{v}=\int_{\Omega}(\nabla \cdot \boldsymbol{\tau}) \mathbf{v}, & \forall \mathbf{v} \in \mathbf{H}_{0}^{1}(\Omega) \\ \int_{\Omega}(\nabla \cdot \boldsymbol{u}) q=0, & \forall q \in L_{0}^{2}(\Omega) \\ \int_{\Omega}\left[\boldsymbol{\tau}+\mathcal{W} e\left(\frac{\partial \boldsymbol{\tau}}{\partial t}+\boldsymbol{u} \cdot \nabla \boldsymbol{\tau}\right)\right]: \mathbf{S}=\int_{\Omega}\left[2 \eta \mathbf{D}+\mathcal{W} e\left(\nabla \boldsymbol{u}^{T} \cdot \boldsymbol{\tau}+\boldsymbol{\tau} \cdot \nabla \boldsymbol{u}\right)\right]: \mathbf{S}, & \forall \mathbf{S} \in \mathcal{S}\end{cases}
$$

\section{Numerical Method}

To solve numerically the problem (6), the so-called reference viscosity scheme was used. The extra stress tensor is split according to $\mathbf{T}=2 \eta \mathbf{D}+\boldsymbol{\tau}$ into the viscoelastic stress $\boldsymbol{\tau}$ and the viscous part $\boldsymbol{\tau}_{s}=2 \eta \mathbf{D}$. The parameter $\eta$ is, in this case, the dimensionless polymer viscosity contribution. This splitting allows to decouple the kinematic and the non-Newtonian viscoelastic stress even though the divergence of $\boldsymbol{\tau}$ is included in the momentum equations as a pseudo-body force while the constitutive equation includes a contribution from the Newtonian part. 
To discretize the momentum equation, the Rothe Method was used where first, the time variable is discretized using a suitable dicretization. The implicit Euler scheme was chosen which approximates the temporal derivative of a function $v$ at time $t_{n}$ by

$$
\frac{\partial v}{\partial t}\left(t_{n}, \cdot\right) \approx \frac{v\left(t_{n}, \cdot\right)-v\left(t_{n-1}, \cdot\right)}{t_{n}-t_{n-1}}
$$

Due to the hyperbolic character of the constitutive equation, the method using characteristics was chosen for discretization. The Characteristic Galerkin Method evaluates time derivatives of vector field on a Lagrangian frame, using the characteristic lines or trajectories describing a material particle when it has been driven by the field at the velocity of the field [13].

The discretization with respect to space employs the mixed finite element method [5], [9], [13] or [14].

\subsection{Semi-descritization}

On the time interval $\left[0, T_{f}\right]$ a set of points (time instants) $t_{n}$ is defined by $t_{n}=n \Delta t=n \frac{T_{f}}{N}$, $n=0, \ldots, N$. The $v^{n}$ denotes the approximation of $v$ at the time instant $t_{n}$, i.e., $v^{n} \approx v\left(t_{n}, \cdot\right)$. Consider $x \in \Omega$.

To discretize the momentum and constitutive equations with respect to time, the implicit Euler scheme was associated with Characteristic Galerkin Method. Hence, the convective time derivative is approximated as:

$$
\frac{D v}{D t}=\frac{\partial v}{\partial t}+\boldsymbol{u} \cdot \nabla v \approx \frac{v^{n}-v^{n-1} \circ X^{n-1}}{d t}
$$

where $X$ is the convecting field defined by $X(x)=x_{t}$ with the particle path $x_{t}$ being the solution of $\dot{x}_{t}=u\left(x_{t}\right), x_{t=0}=x$ and $v \circ X(x)=v(x-u(x) t)=v^{n-1}\left(x^{\star}\right) \equiv v_{\star}^{n-1}$, with $x^{\star}$ the position at time $t_{n-1}$ of the particle located at $x$ at time $t_{n}$.

For the motion equations it leads to:

$$
\begin{cases}\boldsymbol{u}^{0}=u_{0}, & \\ \int_{\Omega} 2(1-\eta) \mathbf{D}^{n}: \nabla \mathbf{v}+\mathcal{R} e \int_{\Omega} \frac{\boldsymbol{u}^{n}-\boldsymbol{u}_{\star}^{n-1}}{\Delta t} \cdot \mathbf{v}-\int_{\Omega} p^{n} \nabla \cdot \mathbf{v}=\int_{\Omega}(\nabla \cdot \boldsymbol{\tau}) \mathbf{v}, & \forall \mathbf{v} \in \mathbf{H}_{0}^{1}(\Omega) \\ \int_{\Omega}\left(\nabla \cdot \boldsymbol{u}^{n}\right) q=0, & \forall q \in L_{0}^{2}(\Omega)\end{cases}
$$

For the constitutive equation it gives:

$$
\int_{\Omega}\left(\boldsymbol{\tau}^{n}+\mathcal{W} e \frac{\boldsymbol{\tau}^{n}-\boldsymbol{\tau}_{\star}^{n-1}}{\Delta t}\right): \mathbf{S}=\int_{\Omega}\left[2 \eta \mathbf{D}+\mathcal{W} e\left(\nabla \boldsymbol{u}^{T} \cdot \boldsymbol{\tau}^{n}+\boldsymbol{\tau}^{n} \cdot \nabla \boldsymbol{u}\right)\right]: \mathbf{S}, \quad \forall \mathbf{S} \in \mathcal{S}
$$

The convergence, stability and other properties of this kind of discretization (7) can be found in literature, e.g. in [9].

\subsection{Finite element method}

The domain $\Omega \subset \mathbb{R}^{2}$ is decomposed into finite number $N$ of triangles $\mathcal{T}$ whose union constitutes a non-degenerated mesh $\mathbb{T}_{h}$. The parameter $h$ is the mesh diameter (maximum cell diameter) of the mesh $\mathbb{T}_{h}$.

The conservation of momentum and mass equations are discretized using the mixed $P_{2}-P_{1}$ finite elements satisfying the compatibility condition known as the discrete LBB (Ladyzheskaya, Babuška and Brezzi) or inf-sup condition, which reads as follows:

There exists $\gamma^{\star}>0$ (independent of $h$ ) such that

$$
\inf _{q_{h} \in M_{h} \backslash\{0\}} \sup _{\boldsymbol{v}_{h} \in \mathbf{X}_{h} \backslash\{0\}} \frac{\left|\left(q_{h}, \nabla^{\prime} \cdot \mathrm{v}_{\mathrm{h}}\right)\right|}{\left\|\mathrm{v}_{\mathbf{h}}\right\|_{\mathbf{X}_{h}}\left\|q_{h}\right\|_{M_{h}}} \geq \gamma^{\star}
$$


where

$$
X_{h}=\left\{\mathbf{v}_{h} \in C(\bar{\Omega}) \cap \mathcal{V}: \mathbf{v}_{\left.h\right|_{\mathcal{T}}} \in \mathbb{P}_{2}(\mathcal{T}), \forall \mathcal{T} \in \mathbb{T}_{h}\right\}, M_{h}=\left\{q_{h} \in C(\bar{\Omega}) \cap L_{0}^{2}(\Omega): q_{h_{\mid \mathcal{T}}} \in \mathbb{P}_{1}(\mathcal{T}), \forall \mathcal{T} \in \mathbb{T}_{h}\right\}
$$

and $\mathbb{P}_{n}$ is the space of polynomials of degree $n>0$ on the triangles, $\mathcal{V}$ and $L_{0}^{2}(\Omega)$ are defined by (3) and (4), respectively.

For the problem (7) and each $h>0$, the approximate finite element problem is defined as:

For each $t \in\left[0, T_{f}\right], u_{0, h} \in X_{h}$ find $\left(\boldsymbol{u}_{h}, p_{h}\right) \equiv\left(u_{h}(t, \cdot), p_{h}(t, \cdot)\right) \in X_{h} \times M_{h}$ such that

$$
\begin{cases}\int_{\Omega} 2(1-\eta) \mathbf{D}_{h}^{n}: \nabla \mathbf{v}_{h}+\mathcal{R} e \int_{\Omega} \frac{\boldsymbol{u}_{h}^{n}-\boldsymbol{u}_{\star, h}^{n-1}}{\Delta t} \cdot \mathbf{v}_{h}-\int_{\Omega} p_{h}^{n} \nabla \cdot \mathbf{v}_{h}=\int_{\Omega}\left(\nabla \cdot \boldsymbol{\tau}_{h}\right) \mathbf{v}_{h}, & \forall \mathbf{v}_{h} \in X_{h} \\ \int_{\Omega}\left(\nabla \cdot \boldsymbol{u}_{h}^{n}\right) q_{h}=0, & \forall q_{h} \in M_{h}\end{cases}
$$

The problem (9) has a unique solution $\left(\boldsymbol{u}_{h}, p_{h}\right) \in X_{h} \times M_{h}$. Moreover,

$$
\lim _{h \rightarrow 0}\left\|u-u_{h}\right\|_{H^{1}(\Omega)}+\lim _{h \rightarrow 0}\left\|p-p_{h}\right\|_{L^{2}(\Omega)}=0
$$

and

$$
\left\|u-u_{h}\right\|_{L^{2}(\Omega)} \leq C(t) h^{2}, \quad\left\|p-p_{h}\right\|_{L^{2}(\Omega)}=C(t) h
$$

Further details about the properties of the above finite element method and the rigorous convergence analysis of spatial discretization of the Navier-Stokes problem can be found in [5].

There is no general mathematical theory to guarantee the stability of finite element method for a given choice of element type, in the case of viscoelastic problems. After some tests for different choices of elements to approximate the tensor, the tensor was approximated by polynomials of the same order as velocity. The finite dimensional space is defined by

$$
\mathcal{S}_{h}=\left\{\mathbf{S}_{h} \in \mathbf{C}(\bar{\Omega}) \cap \mathcal{S}: \mathbf{S}_{h, i j \mid} \in \mathbb{P}_{2}(\mathcal{T}), \forall \mathcal{T} \in \mathbb{T}_{h}\right\}
$$

For the problem (8) and $h>0$, the approximate finite element problem is defined as:

For each $t \in\left[0, T_{f}\right], \boldsymbol{\tau}_{0, h} \in S_{h}$ find $\boldsymbol{\tau}_{h} \equiv \boldsymbol{\tau}_{h}(t, \cdot) \in \mathcal{S}_{h}$ such that

$$
\int_{\Omega}\left(\boldsymbol{\tau}_{h}^{n}+\mathcal{W} e \frac{\boldsymbol{\tau}_{h}^{n}-\boldsymbol{\tau}_{\star, h}^{n-1}}{\Delta t}\right): \mathbf{S}_{h}=\int_{\Omega}\left[2 \eta \mathbf{D}_{h}+\mathcal{W} e\left(\nabla \boldsymbol{u}_{h}^{T} \cdot \boldsymbol{\tau}_{h}^{n}+\boldsymbol{\tau}_{h}^{n} \cdot \nabla \boldsymbol{u}_{h}\right)\right]: \mathbf{S}_{h}, \quad \forall \mathbf{S}_{\mathbf{h}} \in \mathcal{S}_{h}
$$

\subsection{Solution algorithm}

Although the steady solution is searched, the numerical method is based on the unsteady formulation and the time-marching method is employed. It means that the stationary state is attained as the long-time limit solution of the evolutionary model complemented by the steady boundary conditions. If the solution converges in time, the stationary state is obtained. The problem formed by (9), (10) is solved by a decoupled iterative algorithm (Picard method).

The linear system associated to discrete Stokes problem is solved by Crout scheme and the linear system associated to the other equations is solved by a multi-frontal Gauss LU factorization implemented in the FreeFem++ [7], [8], package UMFPACK. 


\subsection{Stabilization technique}

In order to stabilize the simulations for high Weissenberg numbers, a diffusive term was introduced to the constitutive equation. This additional term is defined as

$$
\alpha \cdot \Delta\left(\tau_{h}^{n}-\tau_{h}^{n-1}\right)
$$

It will vanish when $\boldsymbol{\tau}_{h}^{n} \rightarrow \boldsymbol{\tau}$, i.e. the numerical solution will converge to the steady state. The $\alpha$ represents a diffusive parameter of the elastic tensor.

This case is equivalent to take the constitutive equation in the form

$$
\boldsymbol{\tau}+\mathcal{W} e\left(\frac{\partial \boldsymbol{\tau}}{\partial t}+\boldsymbol{u} \cdot \nabla \boldsymbol{\tau}-\nabla \boldsymbol{u}^{T} \cdot \boldsymbol{\tau}-\boldsymbol{\tau} \cdot \nabla \boldsymbol{u}\right)=2 \eta \mathbf{D}+\alpha \Delta(\boldsymbol{\tau}-\boldsymbol{\tau})
$$

for which the weak formulation given by

$\int_{\Omega}\left[\boldsymbol{\tau}+\mathcal{W} e\left(\frac{\partial \boldsymbol{\tau}}{\partial t}+\boldsymbol{u} \cdot \nabla \boldsymbol{\tau}\right)\right]: \mathbf{S}=\int_{\Omega}\left[2 \eta \mathbf{D}+\mathcal{W} e\left(\nabla \boldsymbol{u}^{T} \cdot \boldsymbol{\tau}+\boldsymbol{\tau} \cdot \nabla \boldsymbol{u}\right)\right]: \mathbf{S}-\alpha \nabla(\boldsymbol{\tau}-\boldsymbol{\tau}): \nabla \mathcal{S}, \quad \forall \mathbf{S} \in \mathcal{S}$

Given $\mathcal{M}_{h}=\left\{M_{h} \in C(\bar{\Omega}) \cap L^{2}(\Omega): M_{\left.h\right|_{\mathcal{T}}} \in \mathbb{P}_{2}(\mathcal{T}), \forall \mathcal{T} \in \mathbb{T}_{h}\right\}$, the approximate finite element problem for the tensor can be reformulated as:

For each $t \in\left[0, T_{f}\right], \boldsymbol{\tau}_{0, h} \in S_{h}=\left[\mathcal{M}_{h}\right]^{2 \times 2}$, find $\boldsymbol{\tau}_{h} \in \mathcal{S}_{h}$ with each component $\boldsymbol{\tau}_{h, i j} \equiv \boldsymbol{\tau}_{h, i j}(t, \cdot) \in \mathcal{M}_{h}$, $i, j=1,2$ such that

$\int_{\Omega}\left(\boldsymbol{\tau}_{h, i j}^{n}+\mathcal{W} e \frac{\boldsymbol{\tau}_{h, i j}^{n}-\boldsymbol{\tau}_{\star, h, i j}^{n-1}}{\Delta t}\right): M_{h}=\int_{\Omega}\left[2 \eta \mathbf{D}+\mathcal{W} e\left(\nabla \boldsymbol{u}^{T} \cdot \boldsymbol{\tau}_{h}^{n}+\boldsymbol{\tau}_{h}^{n} \cdot \nabla \boldsymbol{u}\right)\right]_{i j}: M_{h}-\alpha \nabla\left(\boldsymbol{\tau}_{h, i j}^{n}-\boldsymbol{\tau}_{h, i j}^{n-1}\right): \nabla M_{h}$

for all $M_{h} \in \mathcal{M}_{h}$.

\section{Numerical Simulations}

The above described model and algorithm is implemented using the FreeFem $++[7]$ toolboxes. The code was first validated by solving the Poiseuille flow in the straight pipe (2D channel) for which the exact analytical solution is known (this case is not shown here). The numerical simulations presented hereafter are performed for a simple corrugated channel, for which the limits of the code become apparent.

All the tests are performed by considering the dimensionless elastic viscosity parameter $\eta=0.1$. For each fixed $\mathcal{R} e$, the continuation method is applied with respect to $\mathcal{W} e$, which means, the Weissenberg number is increased incrementally, while taking the converged solution obtained for lower $\mathcal{W} e$ as an initial condition for higher $\mathcal{W} e$ simulation.

\section{Boundary conditions}

The inlet and outlet straight parts of the channel allow to consider the flow on the respective boundaries to be fully developed. On the upstream boundary the analytical Poisseuille solution for the fully developed flow in a straight channel for the respective model is imposed. On the solid wall no-slip conditions are used for the fluid velocity.

\section{Artificial diffusion setup}

The initial tests have shown that the diffusive stress algorithm is not only unnecessary for low $\mathcal{W} e$, it may in addition increase the computational time, due to increase of the necessary iterations to steady state. Thus it was decided to only use the stabilization term when necessary, depending on the actual value of the Weissenberg number $\mathcal{W} e$. The artificial diffusion is multiplied by a factor $\alpha(\mathcal{W} e)$ which is vanishing for small Weissenberg number, while it attains some finite asymptotic value for high $\mathcal{W} e$. 


\section{Computational domain}

The corrugated pipe is assumed to consist on straight inlet and outlet parts with dimension. The attached contraction/expansion parts have length $L_{c o n}, L_{\text {exp }}$. Between these parts, several identical segments are inserted. These segments are characterised by its minimum and maximum diameters $D_{\min }$ resp. $D_{\max }$ and the length $L_{\text {seg }}$. All the curvilinear walls have sinusoidal shape.

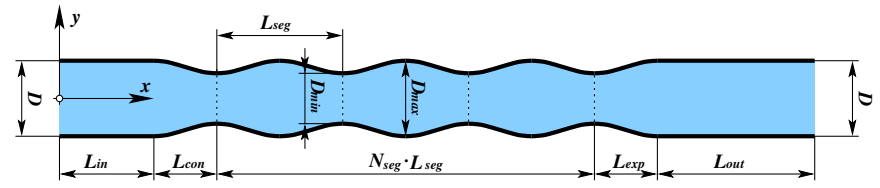

Figure 1: Geometry definition for the corrugated pipe case.

To generate the grids used in the presented simulations, each unit of length on the boundary is first divided into 10 equal segments and then the (almost regular) grid is obtained for the interior of the domain. The grid is generated by FreeFem++, using the Delaunay-Voronoi algorithm.

\subsection{Numerical results}

The above described test case of flow of a viscoelastic fluid through the 2D corrugated channel was solved for a range of Weissenberg and Reynolds number. The aim was to find the limits of the applicability of the original code (without artificial diffusion) and its updated version, using the artificial tensorial diffusion term.

For this particular domain, considering the standard reference viscosity scheme the maximum Weissenberg number reached is 0.4. Using numerical scheme with diffusive stress with variable diffusion parameter the maximum Weissenberg number reached is around 1.0.

The side-by-side comparison of results obtained by both versions of the algorithm for $\mathcal{R} e=400$ and $\mathcal{W} e=0.4$ is possible in the Fig. 2, showing the fields of all computed quantities. The color scales are omitted as only the qualitative comparison of both methods is being discussed. It is obvious, that there are no visible differences between the results provided without or with the artificial diffusive stress.
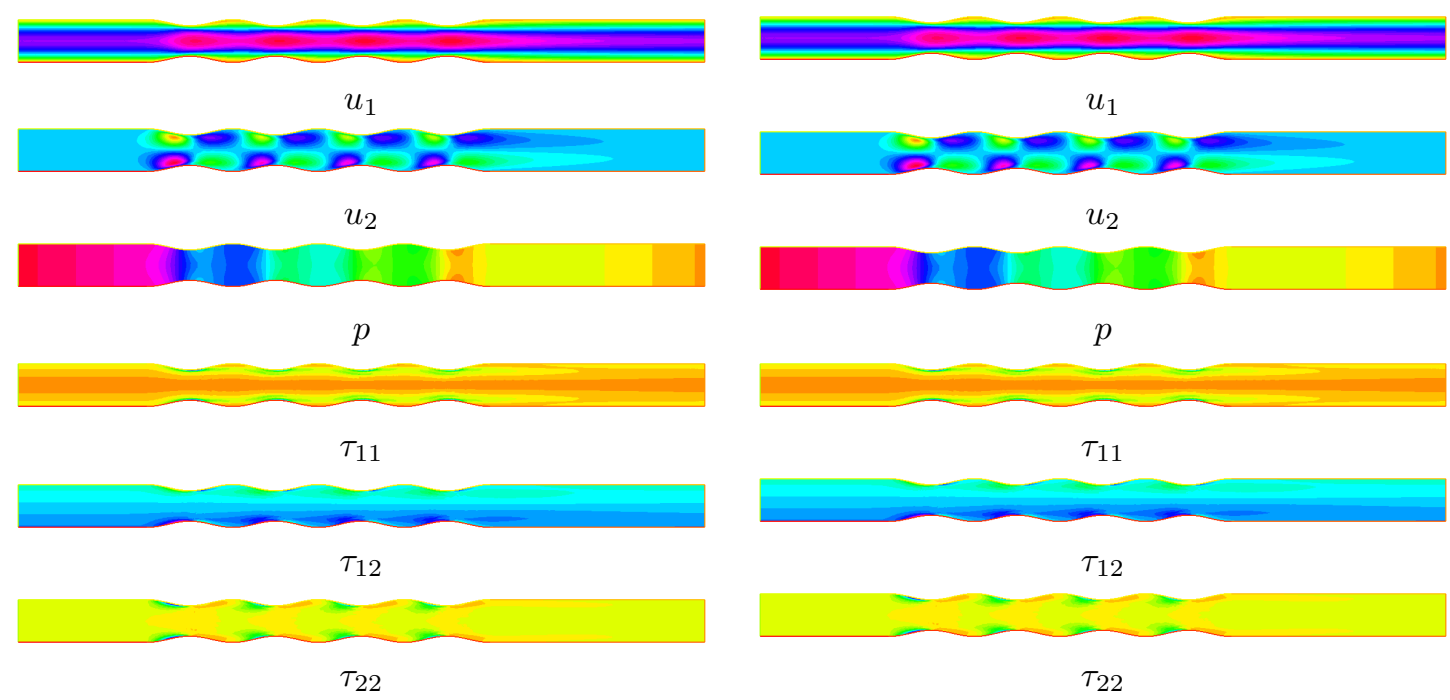

Figure 2: Comparison of components of velocity $u_{1}, u_{2}$, pressure $p$, components of elastic stress tensor $\tau_{11}, \tau_{12}, \tau_{22}$ of Oldroyd-B model for $\mathcal{R} e=400$ and $\mathcal{W} e=0.4$, obtained using the standard algorithm (left) and the algorithm with the diffusive stress (right).

This behavior is not surprising, because the steady state was searched (and found) thus the time derivatives of all quantities vanished and thus also the artificial diffusion term, proportional 
to time-derivative of the stress tensor, has finally disappeared, without having a chance to affect the final solution.

The main advantage and reason to use the artificial tensorial diffusion is that the solution can be obtained also for higher values of the Weissenberg number, where the classical algorithm easily becomes unstable, unable to converge to the steady state.

The extended usability of the algorithm employing the artificial diffusion is possible to see for example in the Fig. 3-4, where the pressure drop (i.e. the difference between outlet and inlet pressure) is shown for both versions of the algorithm for a whole range of Weissenberg and Reynolds numbers.

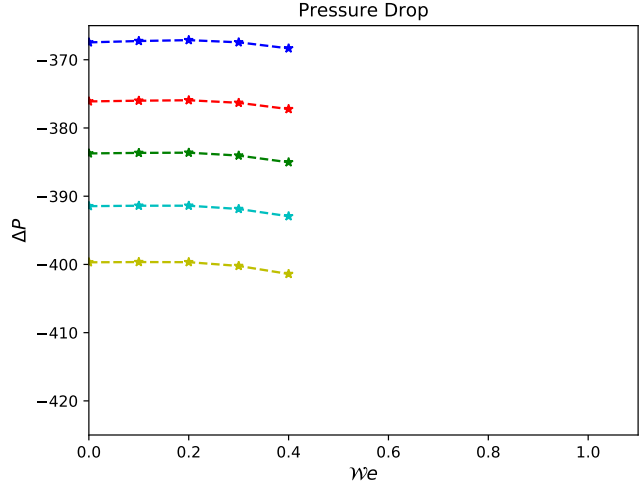

(a) without diffusion

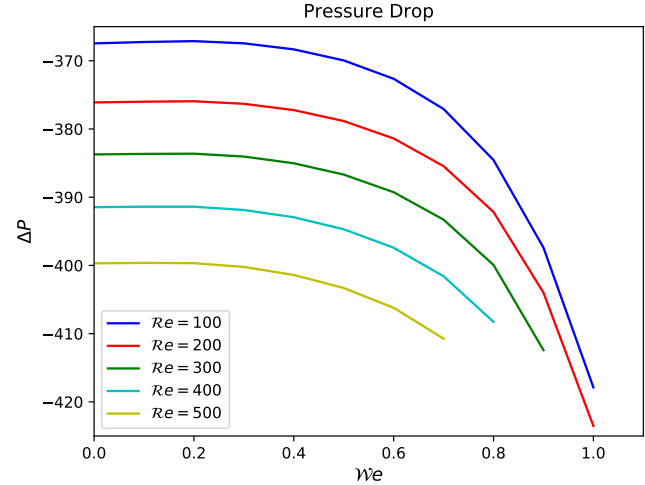

(b) with diffusion

Figure 3: Pressure drop for different Reynolds and Weissenberg numbers, obtained with and without the artificial stress diffusion.

Not only that there is a visible increase in the maximum attainable Weissenberg number when the artificial diffusion is employed, but as before, the solutions are identical in the range where both algorithms converge. This agreement between the two algorithms is apparent in the Fig. 4, where both solutions are plotted into one figure. Again, the continuous line is reserved for the diffused algorithm and the asterisk for the non-diffused algorithm.

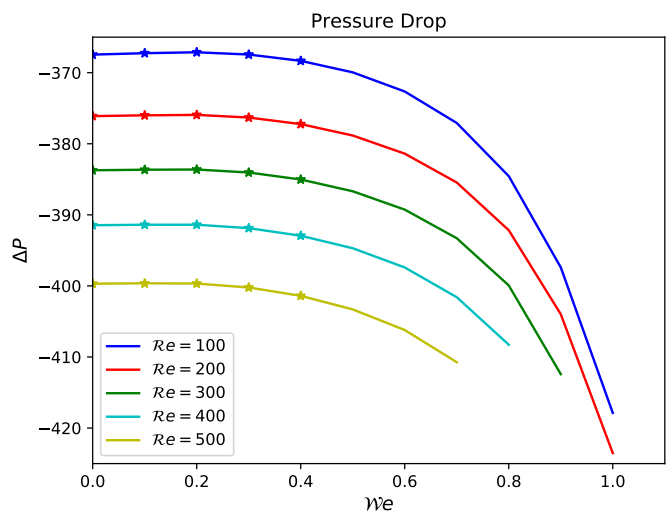

Figure 4: Comparision of the pressure drop for different Reynolds and Weissenberg numbers, obtained with and without the artificial stress diffusion (superposition of the plots above).

Obviously, both algorithms provide identical solutions up to the $\mathcal{W} e=0.4$, above which the non-diffusive algorithm fails to converge, while the one with artificial diffusion keeps providing stable solution up to $\mathcal{W} e \approx 1.0$. The limit value obviously depends on the Reynolds number of the particular case. 


\section{Conclusions and Remarks}

The presented algorithm and solution code was successfully implemented using FreeFem ++ package. It was shown that the original formulation of the numerical method becomes unstable (for given computational test case) for Weissenberg number $\mathcal{W} e>0.4$. The numerical stability and robustness of the code can be significantly improved by using an artificial diffusive stress term in the form (11), (12) respectively. This extra diffusive term allowed to obtain the solution of the given problem up to $\mathcal{W} e \approx 1.0$, with the limit value slightly depending on the Reynolds number.

Future investigation will focus on the verification of this type of artificial diffusive term when using other numerical methods, namely the finite volumes and finite differences. Also the question of the use of this stabilization technique for unsteady problems remains open.

\section{Acknowledgment}

The financial support for the present project was partly provided by the Fundação para a Ciência e a Tecnologia - FCT under the Grant Ref. SFRH/BSAB/150464/2019 and partly by the Czech Science Foundation under the grant No. P201-19-04243S.

\section{References}

[1] Arada, N., Pires, M., \& Sequeira, A.: Numerical simulations of a shear-thinning Oldroyd-B fluids in curved pipes.IASME Transactions, Vol. 2, no. 6: (2005) pp. 948-959.

[2] Arada, N., Pires, M., \& Sequeira, A.: Numerical approximation of a viscoelastic Oldroyd-B flows in curved pipes. Kyoto Conference on the Navier-Stokes Equations and their Applications. RIMS Kôkyûroku Bessatsu, vol. B1: (2007) pp. 43-70.

[3] Arada, N., Pires, M., \& Sequeira, A.: Viscosity effects on flows of generalized Newtonian fluids through curved pipes. Computers and Mathematics with Applications. Vol. 53: (2007) pp. 625-646.

[4] Bodnár, T., Pires, M. \& Janela, J.: Blood flow simulation using traceless variant of JohnsonSegalman viscoelastic model. Math. Model. Nat. Phenom. Vol. 9, no. 6: (2014) pp. 117-141.

[5] Girault, V. \& Raviart, P.-A.: Finite Element Methods for the Navier-Stokes Equations. Springer, Heidelberg(1986).

[6] Groisman, A. \& Steinberg, V.: Mechanism of elastic instability in Couette flow of polymer solutions. Experiment Phys. Fluids, vol. 10: (1998) pp. 2451-2463.

[7] Hecht, F.: New development in FreeFem++. Journal of numerical mathematics, vol. 20, no. 34: (2012) pp. 251-266.

[8] Hecht, F. \& Danaila, I.:FreeFem++ Lessons 5-8. https://www.ljll.math.upmc.fr/ hecht/ftp/ff++/2016-Fields/FH-Lesson5-8-Fields.pdf (2016).

[9] Marion, M. \& Temam, R.: Navier-Stokes Equations: Theory and Approximation. Handbook of Numerical Analysis vol. VI: (1998).

[10] Oldroyd, J.: Non-Newtonian effects in steady motion of some idealized elasto-viscous liquids. Proc. R. Soc. A. 245: (1958) pp. 278-297.

[11] Owens, R. \& Phillips, T. : Computational Rheology. Imperial College Press, London (2002).

[12] Pires, M. \& Sequeira, A.: Flows of Generalized Oldroyd-B Fluids in Curved Pipes. In: Escher J. et al. (eds) Parabolic Problems. Progress in Nonlinear Differential Equations and Their Applications, Springer, Basel, Vol. 80: (2011) pp. 21-43.

[13] Quarteroni, A. \& Valli, A.: Numerical Approximation of Partial Differential Equations Springer Publishing Company, Incorporated 2nd ed (2008).

[14] Rannacher, R.: Finite Element Methods for the Incompressible Navier-Stokes Equations. Lecture notes (1999). 